\title{
Abordaje intervencionista del síndrome facetario lumbar: Denervación con radiofrecuencia
}

\author{
Alberto Martínez-Martínez', Mgtr; J. García-Espinosa', Mgtr; F. Ruiz-Santiago', PhD; L. Guzmán-Álvarez', PhD; M \\ Castellano-García', Mgtr.
}

1. Unidad de Gestión Clínica de Radiodiagnóstico, Hospital Campus de la Salud, Complejo Hospitalario Universitario de Granada, España.

\section{Interventional approach for lumbar facet syndrome: Radio frequency denervation}

\begin{abstract}
Lumbar pain is a common and frequent chronic condition. It is estimated that facet joint pain represents $15-31 \%$ of all consultations for low back pain. In suspected facet joint pain, it is recommended to perform nerve blocks to establish the diagnosis. Percutaneous continuous radiofrequency is the most effective treatment to date. We describe the current evidence on the minimally invasive treatment of lumbar facet syndrome and our experience with image guidance with CT and fluoroscopy by means of rhizolysis of the medial branch of the dorsal primary ramus of the spinal nerve.
\end{abstract}

Keywords: Low back pain, Radiofrequency treatment, Nerve block.

Resumen. El dolor lumbar es una entidad común y frecuente crónica. Se estima que el dolor de causa articular facetaria representa el 15-31\% de todas las consultas por dolor lumbar. En sospecha de dolor articular facetario se recomienda realización de bloqueos nerviosos para establecer el diagnóstico. La radiofrecuencia continua percutánea es el tratamiento más efectivo hasta la fecha. Se describe la evidencia actual en el tratamiento mínimamente invasivo del síndrome facetario lumbar y nuestra experiencia con las guías de imagen con TC y fluoroscopía mediante rizolisis de la rama medial del ramo primario dorsal del nervio espinal.

Palabras clave: Dolor lumbar, Tratamiento con radiofrecuencia, Bloqueo nervioso.

Martínez A. et.al. Abordaje intervencionista del síndrome facetario lumbar. Rev Chil Radiol 2017; 23(1): 7-14. Correspondencia: Alberto Martínez-Martínez / rralbert.martinez@gmail.com

Trabajo recibido el 27 de agosto de 2016. Aceptado para publicación el 17 de marzo de 2017.

\section{Introducción}

El dolor de espalda, en especial en columna lumbar, afecta a un alto porcentaje de la población y presenta un significativo impacto socioeconómico, con altas tasas de ausentismo y bajas laborales. En torno al $70-80 \%$ de la población de países desarrollados padece lumbalgia o lumbociatalgia en alguna ocasión a lo largo de su vida, constituyendo el motivo de consulta más frecuente tanto en atención primaria como en otras especialidades como rehabilitación, cirugía ortopédica y reumatología. En los Estados Unidos la prevalencia de dolor crónico de espalda es superior al $10 \%$. Hasta un cuarto de la población sufre al menos un episodio de dolor lumbar al año $0^{1-5}$.

El síndrome facetario lumbar se encuentra dentro de las etiologías estructurales de dolor de espalda. Su prevalencia como causa de dolor lumbar está entre el $15-31 \%^{3}$ con incidencia a lo largo de la vida de hasta el $52 \%$ en algunas series ${ }^{6-10}$. Su causa es la degeneración de las articulaciones facetarias, las cuales sufren los cambios propios de cualquier articulación sinovial. Clínicamente cursa con dolor lumbar de características mecánicas que aumenta con los movimientos de rotación y flexo-extensión (en especial en hiperextensión). No es rara su irradiación a nalgas y cara posterior de muslos. El comienzo y el curso suelen ser insidiosos, aunque muchos pacientes lo refieren a raíz de un esfuerzo brusco'.

Actualmente no existen criterios diagnósticos aceptados del síndrome facetario. Debe valorarse 
la posibilidad de una espondiloartropatía inflamatoria para lo cual es indispensable un adecuado estudio clínico y analítico. Las técnicas de imagen son de utilidad para descartar otras entidades, aunque sus hallazgos no son específicos. La radiografía simple, Resonancia Magnética (RM) y Tomografía Computarizada (TC) pueden mostrar hallazgos de degeneración facetaria (degeneración del cartílago, esclerosis, osteofitos, quistes sinoviales), los cuales no se suelen correlacionar con la intensidad del dolor. Algunos trabajos han demostrado la existencia de hallazgos de degeneración facetaria en TC y RM sin sintomatología asociada ${ }^{11}$.

En la actualidad, el estándar para el diagnóstico del síndrome facetario lumbar es el bloqueo de la rama medial del ramo dorsal del nervio espinal, el cual aporta inervación sensitiva a las articulaciones interapofisarias. Está aceptado que el único medio diagnóstico del síndrome facetario es el bloqueo nervioso, no debiendo existir discusión, según la evidencia disponible, sobre la necesidad de su realización para establecer el diagnóstico de síndrome facetario antes de llevar a cabo técnicas ablativas nerviosas ${ }^{8,12-13}$.

El tratamiento del síndrome facetario está fundamentado en un abordaje multimodal y multidisciplinar que combina las terapias conservadoras, incluidos los tratamientos orales farmacológicos, con las terapias mínimamente invasivas. Hay menos evidencias de la utilidad de las terapias conservadoras en su manejo, las cuales únicamente parecen tener utilidad como terapias adyuvantes ${ }^{15-16}$

Actualmente el abordaje estándar de tratamiento consiste en bloqueos nerviosos y rizolisis o neurolisis de la rama medial del ramo dorsal del nervio espinal8-14.

\section{Evidencias históricas y actuales del tratamiento del síndrome facetario}

Es bien conocida, desde el año 1933 a raíz de los trabajos de Ghormerly, la ausencia de una clara correlación entre la artrosis facetaria en técnicas de imagen y el dolor lumbar. La prevalencia de la artrosis facetaria llega a alcanzar en algunas series el $89,2 \%$ en personas de entre 60-69 años, siendo menos prevalente el cuadro clínico de síndrome facetario lumbar ${ }^{14-7}$. En 1963 Hirsch demostró que, a pesar de la poca asociación entre los cambios degenerativos facetarios y el cuadro clínico, existía relación entre las articulaciones facetarias y el hoy conocido como síndrome facetario, ya que la introducción de suero fisiológico hipertónico intra articular reproducía el dolor de espalda típico del síndrome facetario ${ }^{18}$. En los años 70, Rees y colaboradores, realizaron las primeras rizolisis con éxito de articulaciones facetarias cervicales y lumbares ${ }^{19-20}$.

A pesar de su uso extendido en la práctica clínica, incluso en la actualidad, ya se demostró el poco valor diagnóstico y terapéutico de las inyecciones intra articulares de anestésico y corticoides ${ }^{21-22}$.

Actualmente está extensamente demostrada la utilidad del bloqueo nervioso sin y con corticoides para el tratamiento y en especial para el diagnóstico del síndrome facetario ${ }^{14,23-25}$.

A pesar de la disociación clínico radiológica entre la artrosis facetaria y el síndrome clínico, está más que aceptada la etiología degenerativa facetaria como causa del síndrome facetario.

A lo largo de los años ha ido en aumento la evidencia en el uso de la radiofrecuencia para el tratamiento del síndrome facetario mediante la rizolisis de la rama medial del ramo dorsal del nervio espinal. Debido al empleo de distintos tipos de radiofrecuencia, pulsada (RFp) o continua (RFc); diferentes técnicas empleadas y diversos criterios de selección de los pacientes, la tasa de éxito varía en la literatura desde sin efecto a muy efectiva. La revisión sistemática más importante hasta la fecha es la de Falco et al. ${ }^{26}$ en la que se incluyen 122 estudios (11 ensayos clínicos) de la que se puede concluir que hay buena evidencia para el uso de RFc y evidencia limitada para la RFp.

\section{Radiofrecuencia}

El término radiofrecuencia (RF) hace referencia a la porción menos energética del espectro electromagnético. Es un tipo de onda o radiación electromagnética con alta frecuencia $(400-500 \mathrm{~Hz})$ que se encuentra en la categoría de radiaciones no ionizantes. Sus primeras aplicaciones con fines médicos datan de 1931, cuando Kirschner la usó por primera vez para tratar una neuralgia del trigémino ${ }^{27}$. Existen varios sistemas y formas de aplicaciones de radiofrecuencia con fines terapéuticos ${ }^{28}$. Los sistemas de aplicación de uso más extendido son los monopolares. En ellos se debe colocar al paciente un electrodo neutro o de dispersión (cojinete de tierra) que se sitúa en el muslo o brazo del paciente. El paciente forma parte de un circuito cerrado constituido por el generador de radiofrecuencia, la corriente de entrada a través del electrodo y la corriente de salida que se recoge en el electrodo de dispersión (Figura 1). El voltaje viene determinado por la diferencia de potencial entre el electrodo de entrada y el receptor. El sistema monopolar es el de uso más extendido en la rizolisis ${ }^{29}$.

Además de los distintos sistemas de radiofrecuencia hay diferentes formas de aplicarla. La RFp se administra mediante pulsos breves de RF de alto voltaje, pudiendo alcanzar temperaturas en torno a 40-42 ${ }^{\circ}$, con disipación del calor entre pulsos evitando la coagulación del tejido. El tipo de efecto que presenta en el tejido es por tanto poco destructivo presentando actividad principalmente neuromoduladora aunque su mecanismo de acción es aún desconocido. La $\mathrm{RFc}$, también llamada térmica o convencional, se administra como su propio nombre indica de forma 


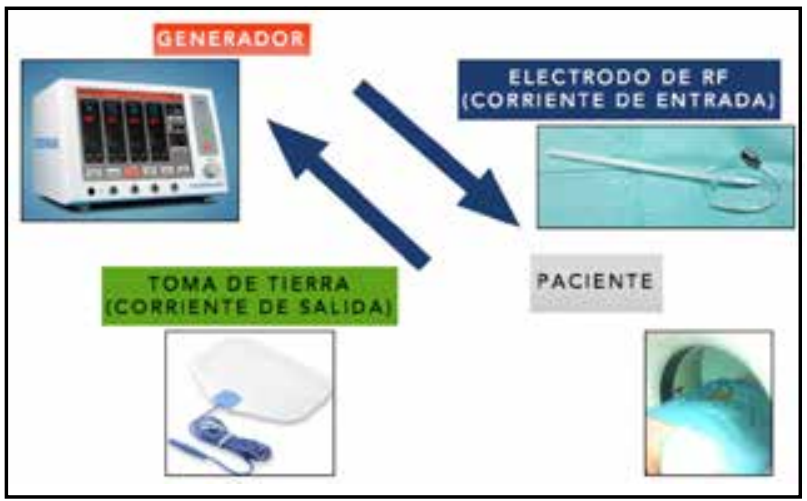

Figura 1. Componentes del circuito de radiofrecuencia monopolar.

continua, sin periodos en los que se disipe el calor y con temperaturas de hasta $80-82^{\circ}$. Su efecto biológico consiste en la destrucción del tejido sobre el que se aplica, ya que a partir de $50-55^{\circ} \mathrm{C}$ el daño celular es irreversible $e^{6,29}$.

\section{Anatomía facetaria}

La importancia de la rama medial del ramo dorsal del nervio espinal radica en que aporta inervación sensitiva a las articulaciones facetarias. Los ramos dorsales lumbares emergen desde su foramen de conjunción correspondiente (L1 entre L1-L2; L2 entre L2-L3; etc). El ramo dorsal del nervio espinal tiene una rama lateral, una rama intermedia y una rama medial que se dirige posterior y caudalmente pasando por encima de la apófisis transversa de la vértebra y bajo el ligamento mamilo accesorio, abrazando el cuello de la apófisis articular superior para dar finalmente ramas al músculo multífido, interespinoso, ligamentos amarillos y la faceta superior e inferior (Figura 2). La raíz L5 tiene una particularidad, ya que su rama medial del ramo dorsal no discurre sobre ninguna apófisis transversa, sino sobre la escotadura existente entre el ala sacra y la apófisis articular superior de S1 (Figura 3). Conocer la relación de las ramas mediales con la apófisis tranversa es, ya que es en esta localización donde realiza el bloqueo o la posterior neurolisis.

La inervación facetaria es dual, es decir, cada faceta tiene una inervación por dos ramos mediales, por lo que debemos actuar sobre ambos para denervar completamente una articulación (Figura 4) 7,8,30 $^{7}$. A efectos prácticos, en un paciente con un claro síndrome facetario con nivel L4-L5, se realizará el procedimiento sobre las apófisis transversas de L4 y L5. De esta forma habremos actuado sobre la rama medial del ramo dorsal de L3 y L4 que transcurren sobre las apófisis transversas de L4 y L5 respectivamente.

En nuestro centro, salvo en caso de existencia de un claro nivel en la exploración física, realizamos la ablación en los niveles vertebrales L4, L5 y S1, por lo que denervamos de forma completa las articula-

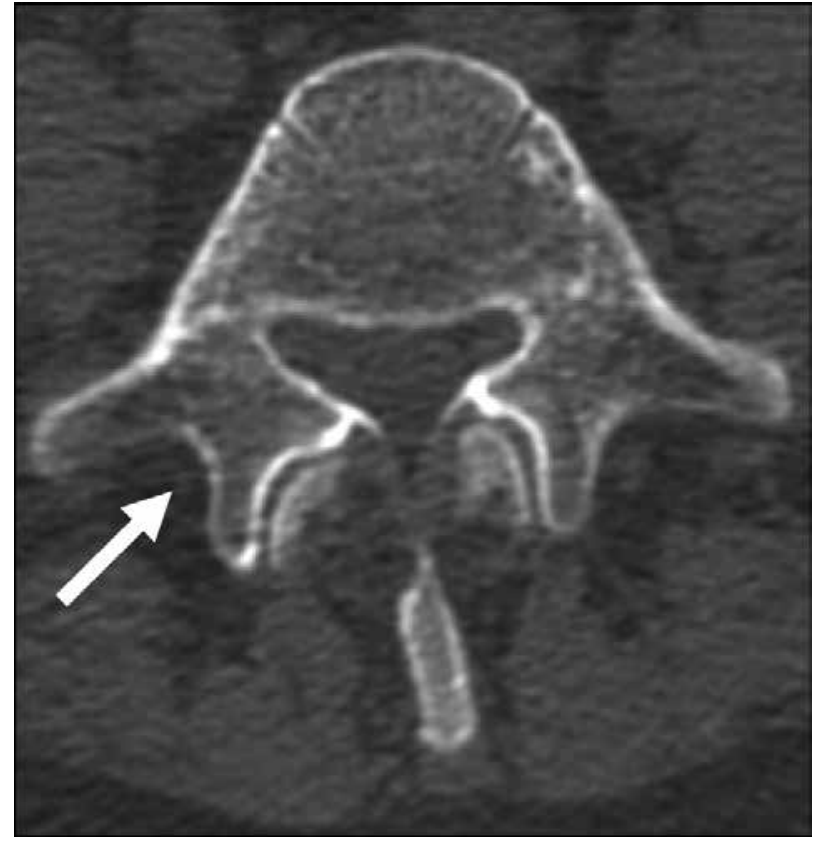

Figura 2. Imagen axial de TC en donde se sitúa la teórica localización de la rama medial del ramo dorsal del nervio espinal (flecha).

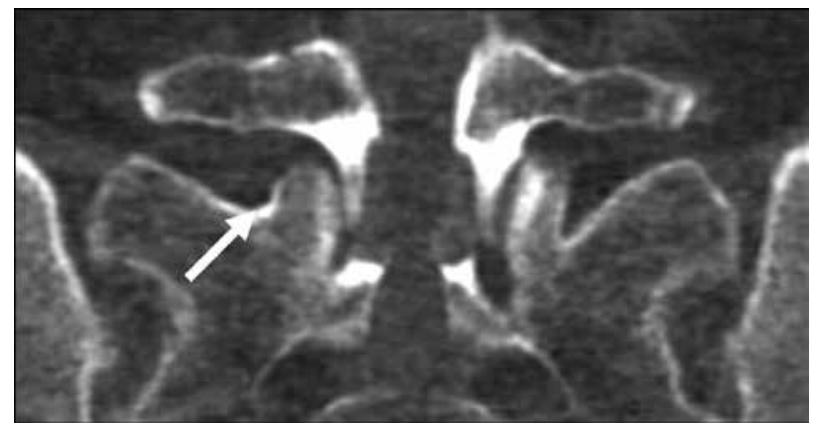

Figura 3. Imagen coronal oblicua de TC que muestra la teórica localización de la rama medial del ramo dorsal del nervio espinal L5 (flecha), situada en la escotadura entre el ala sacra y la apófisis articular superior.

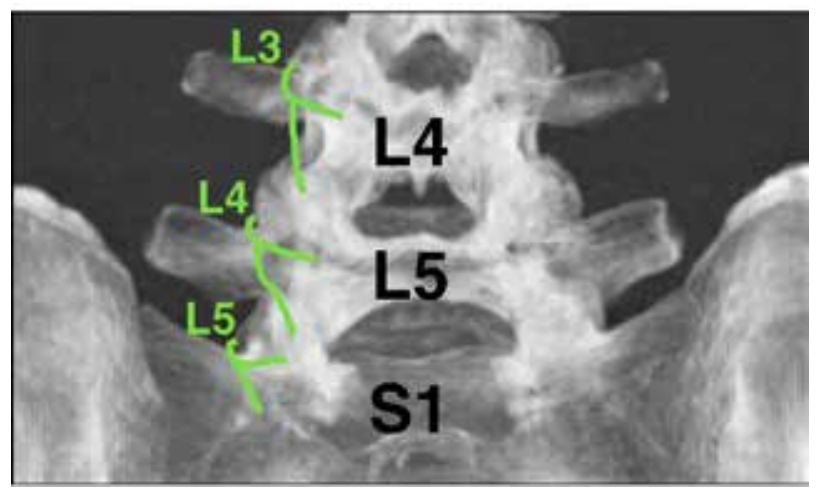

Figura 4. Esquema de la inervación dual facetaria a nivel L4-S1 en el que se aprecian las ramas mediales de los ramos dorsales encargados de su inervación sensitiva. 
ciones L4-L5 y L5-S1 que son las más implicadas en el síndrome facetario.

\section{Bloqueo nervioso facetario}

El bloqueo de la articulación interapofisaria tiene un objetivo diagnóstico y terapéutico, ya que nos determinará con su efectividad, si el síndrome facetario es la causa fundamental del dolor que presenta el paciente ${ }^{2}$. El bloqueo se ha definido como una técnica más sencilla y segura que la infiltración intra articular de esteroides quedando esta última relegada a pacientes con evidente esclerosis y contraindicación de una posterior $\mathrm{RF}^{14,25}$. Actualmente la ASIPP (American Society of Interventional Pain Physicians) recomienda el uso del bloqueo diagnóstico, siempre bajo control de imagen, en pacientes con sospecha clínica de dolor lumbar de origen facetario 2,14.

La técnica para su realización es similar a la de la $\mathrm{RF}^{6,7}$, que se describirá posteriormente.

Un bloqueo se define como positivo cuando existe: ${ }^{14,29,31}$

- Disminución $50 \%-74 \%$ del dolor basal en al menos un test (pobre evidencia) y dos test (evidencia suficiente).

- Disminución $>75 \%$ en un test (evidencia limitada).

- Disminución $>75 \%$ en dos test (mejor evidencia).

La existencia de un test de bloqueo positivo, preferiblemente al menos en el grado de evidencia limitada, es un paso indispensable para la adecuada selección de los pacientes de cara a la posterior rizolisis. No está definido en las guías clínicas ni en revisiones sistemáticas un mínimo temporal de respuesta positiva al bloqueo ${ }^{14}$.

\section{Indicaciones y contraindicaciones de la $\mathbf{R F}^{1,2,4-}$} 9,12-4,23-26

\section{Selección de pacientes}

- Dolor lumbar crónico clínicamente compatible, con fracaso del tratamiento conservador.

- Correcta evaluación clínica, incluyendo factores psicosociales (los trastornos del ánimo se han asociado con un peor pronóstico).

- Test de bloqueo positivo: de forma ideal con respuesta $>75 \%$ en dos test.

- Difícil seleccionar pacientes con síndrome de cirugía fallida de espalda (síndrome postlaminectomía), en los cuales sólo un 3\% obedecen a causa facetaria como generadora de su sintomatología.

- Evaluación de hallazgos en imagen:

- Pueden existir hallazgos que ayuden a confirmar el síndrome facetario como son los cambios degenerativos facetarios, aunque no es un factor indispensable.
- Hallazgos que descarten el síndrome facetario: discopatía con clínica concordante, hallazgos propios de espondiloartropatías seronegativas, espondilodiscitis.

\section{Exclusión de pacientes}

- Pacientes con un claro dolor radicular (la irradiación tiende a alcanzar el pie), claudicación neurógena (que obligará a descartar estenosis de canal), déficits neurológicos motores.

- Enfermedad médica aguda o no controlada, reacción alérgica a la anestesia local, embarazo o lactancia.

- Marcapasos cardiaco: no sería una contraindicación absoluta si se realiza radiofrecuencia bipolar lejos del marcapasos o monopolar colocando el electrodo de dispersión en muslo en lugar de en brazo.

\section{Procedimiento}

Antes de iniciar el procedimiento debe efectuarse una anamnesis para detectar contraindicaciones que no se hayan recogido en la historia clínica del paciente. Es conveniente realizar previamente algún test para la valoración del dolor. En nuestra unidad determinamos la puntuación en la Escala Visual Analógica del dolor (EVA) y realizamos un test de Oswestry.

En condiciones ideales es posible que el paciente defina perfectamente el nivel vertebral sintomático, siendo su clínica reproducible con la compresión vertebral local. En este caso se podría colocar en la piel algún marcador radio opaco y denervar selectivamente el nivel sintomático. En la práctica clínica el dolor suele ser más difuso en región lumbar baja, por lo que en nuestro centro realizamos los niveles vertebrales L4, L5 y S1; con los que denervaremos de forma completa las articulaciones L4-L5 y L5-S1. Todo paciente debe disponer de una vía venosa periférica antes de la realización de la intervención.

La colocación del paciente, independientemente de la técnica de imagen a emplear, será en decúbito prono con una almohada, sábanas o colchón bajo el abdomen para disminuir la lordosis lumbar del paciente, garantizando su comodidad. Se deben mantener unas condiciones lo más asépticas posibles. No es recomendable el uso de analgesia en el test de bloqueo facetario debido a los falsos positivos que se pueden ocasionar. El uso de benzodiacepinas puede ser necesario en determinados pacientes ${ }^{7-9,12,13,16,30-37}$.

\section{a) Técnicas de imagen como guía del procedimiento}

Radioscopia o Fluoroscopia

Históricamente y aún en la actualidad, la técnica de imagen más empleada para la realización de bloqueos y rizolisis, es la radioscopia o fluoroscopía. La "diana" u objetivo es la unión de la apófisis transversa con la apófisis articular superior, ya que es el sitio 
por el que discurre la rama medial del ramo dorsal del nervio espinal antes de dar sus ramas superior, posterior e inferior.

Existen varios tipos de abordaje fluoroscópico:

\section{- Abordaje oblicuo}

Con el paciente en decúbito supino se centra el tubo de rayos $X$ con una leve angulación caudal $\left(10-15^{\circ}\right)$ para que desaparezca el doble contorno del margen inferior de los cuerpos vertebrales. Desde esa posición posteroanterior (PA) se angula el tubo unos $25-30^{\circ}$ hacia el lado a intervenir obteniendo una proyección oblicua de la columna en la que se visualiza el típico "perrito escocés". Debemos identificar la unión del "hocico" (apófisis transversa) con el "ojo" (pedículo) y la "oreja" (apófisis articular superior) (Figura 5A). La punción deberá iniciarse más lateral al objetivo y desde ahí medializar la aguja. Es recomendable este abordaje (más paralelo al nervio) en lugar del perpendicular, ya que así se consigue un mayor contacto de la punta activa del electrodo con el nervio (técnica de Norteamérica).

\section{- Abordaje PA}

Con el paciente en decúbito supino se angula el tubo de rayos $x$ caudalmente unos $10-15^{\circ}$. Se identifica la apófisis transversa y el pedículo. Con la técnica de Norteamérica (de lateral a medial) se dirige la punta hacia la diana hasta contactar con la porción más medial y craneal de la apófisis transversa (Figura 5B).

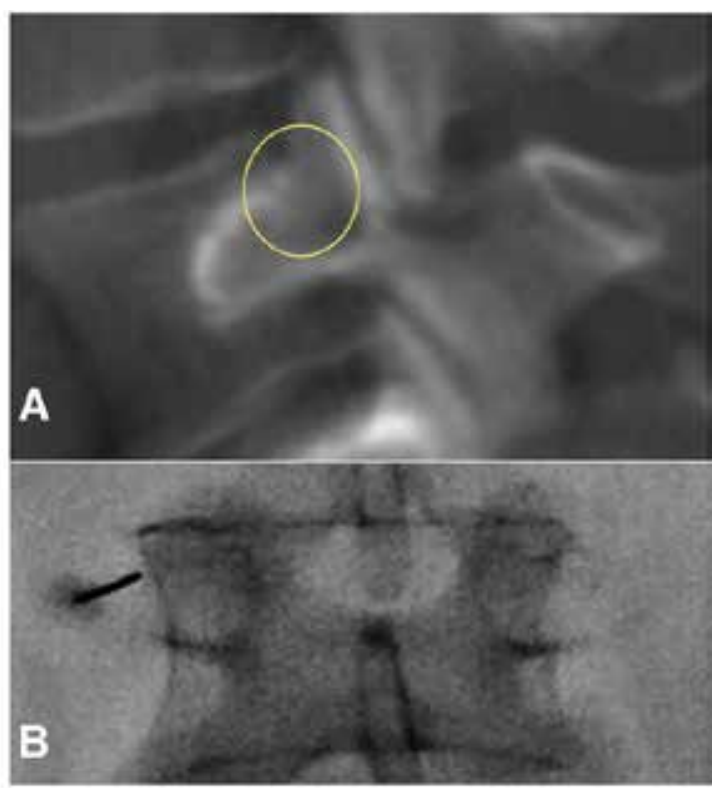

Figura 5. Imagen fluoroscópica oblicua $(A)$ en donde se evidencia la imagen de "perrito escocés" con la teórica localización de la rama medial del ramo dorsal del nervio espinal (círculo). Imagen fluoroscópica posteroanterior (B) con aguja situada de forma correcta en la porción más superior de la apófisis transversa en la localización de la rama medial del ramo dorsal.
$\underline{\mathrm{TC}}$

El uso de la guía con TC está mucho menos extendido en la práctica clínica, existiendo escasos estudios en la literatura ${ }^{32-35}$. En el único estudio aleatorizado realizado hasta la fecha comparando fluoroscopia y TC, esta última ha ofrecido mejores resultados en cuanto a menor puntuación de EVA al mes y tres meses postratamiento aunque no de forma estadísticamente significativa ${ }^{35}$.

La colocación del paciente es similar a la de la guía radioscópica. En nuestro centro, a la hora de la realización del TC para planificar la punción, utilizamos una "rejilla" a modo de guía radio opaca situada sobre la piel del paciente, la cual nos permitirá establecer el punto de entrada de la aguja en el eje X, pudiendo situar el eje Z mediante el marcador láser del scanner de TC (Figura 6). Los abordajes posibles de la "diana" son de forma perpendicular u oblicua. Aunque no existe en la actualidad en la literatura una comparación de ambas aproximaciones con TC, hemos observado resultados favorables al abordaje oblicuo (técnica de Norteamérica) tanto en los test de estimulación sensitiva y motora como en la mejora de la escala de EVA.

Una vez marcados los puntos de punción en la piel del paciente se establece un campo estéril con cualquier antiséptico adecuado. En operadores con experiencia es posible avanzar la aguja de forma directa hasta contactar con el objetivo manteniendo el ángulo de oblicuidad establecido, no obstante, es conveniente la realización de controles de TC con reducción de la dosis para comprobar el adecuado avance y la correcta localización final de la aguja.

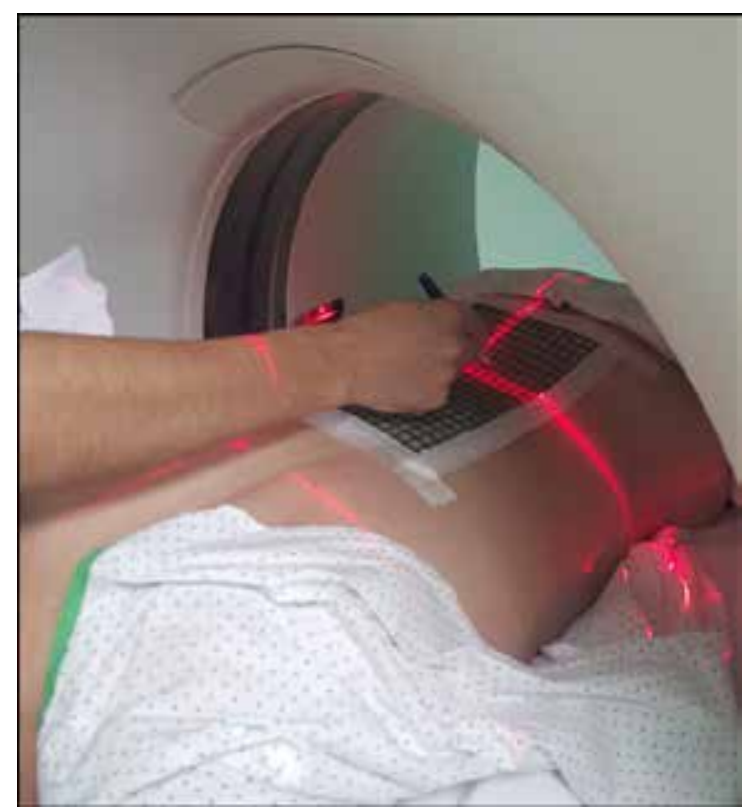

Figura 6. Señalización de los puntos de punción con la referencia de la rejilla radio opaca para establecer el eje $X$ y la marca láser del scanner de TC para el eje $Z$. Tras señalizar los puntos con rotulador permanente se puede retirar la rejilla. 


\section{b) Aplicación de la radiofrecuencia}

Confirmada la localización adecuada de la punta del electrodo es fundamental realizar un test de estimulación sensorial y motora. La gran mayoría de dispositivos de RF comercializados en la actualidad disponen de programas con los parámetros pre establecidos para la realización del test de estimulación y la posterior aplicación de la RF.

- Estimulación sensorial $(50 \mathrm{~Hz})$ : el paciente notará una sensación de parestesias, presión o molestia leve en su área dolorosa con un voltaje igual o inferior a $0,5 \mathrm{v}$.

- Estimulación motora (2Hz): deberá ser positiva a voltajes no inferiores a 1,5-2 veces el voltaje obtenido en la estimulación sensorial (punto de disociación sensitivo-motora). Se manifiesta como una fasciculación de la musculatura paravertebral, no debiendo aparecer estimulación radicular del miembro inferior. Un test motor positivo a un voltaje menor al del punto de disociación sensitivo-motora indica una situación muy próxima a nervios motores y la necesidad de re-situar la punta del electrodo. Como ejemplo, un test sensorial con valor $0,4 \mathrm{v}$ no debería presentar un test motor positivo con valores inferiores a 0,2 v. Una vez que los electrodos estén correctamente situados (Figura 7) se debe anestesiar la zona antes de la aplicación de la radiofrecuencia. En nuestro centro empleamos una mezcla de $10 \mathrm{ml}$ de mepivacaina al $1 \%$ con $80 \mathrm{mg}$ $(2 \mathrm{ml})$ de acetónido de triamcinolona que distribuimos de forma bilateral en los niveles L4, L5 y S1. Otros autores usan únicamente anestésico local con distintas presentaciones y diluciones. A continuación, se inicia la rizolisis mediante un programa automático de RFc con la temperatura máxima fijada a $8^{\circ} \mathrm{C}$ (pudiendo alcanzar temperaturas de hasta $81-82^{\circ}$, ya que es el propio tejido el que calienta la punta del electrodo) y duración de 90 segundos. Finalizado el procedimiento el paciente es observado aproximadamente durante una hora para valorar la aparición de incidencias inmediatas, incorporándose a su vida normal con la indicación de no realizar ejercicios bruscos en 2-3 semanas ${ }^{7-9,12-14,30-36}$. c) Complicaciones y efectos secundarios del bloqueo nervioso y radiofrecuencia ${ }^{7,8,11,14,16,26,37}$

- Sangrado: hematoma de partes blandas, epidural, medular o perirradicular.

- Infección: absceso de partes blandas, epidural o facetario; meningoencefalitis.

- Trauma: punción dural inadvertida.

- Inyección subdural, epidural, foraminal o intravascular del corticoide

- Corticoides: supresión del eje hipofisariosuprarrenal, hipocortisolismo, Cushing, osteoporosis, necrosis ósea avascular, miopatía esteroidea, lipomatosis epidural, aumento de peso, hiperglucemia.

d) Complicaciones e inconvenientes propios de la radiofrecuencia ${ }^{7,8,11,14,16,26,37}$

- Ablación yatrogénica radicular, de la rama motora o incluso medular (raro con la guía TC).

- No es raro que se produzca un empeoramiento del dolor basal tras RF, que será autolimitado y que puede prevenirse en parte con la infiltración de corticoides en el mismo procedimiento.

- Neuralgia postdenervación: quemazón o disestesias que duran en torno a 6-8 semanas.

- Disminución de la sensibilidad cutánea (por ablación de ramas cutáneas).

- Dolor transitorio en piernas o debilidad permanente.

Si bien no se considera complicación, uno de los principales inconvenientes de la radiofrecuencia es la reaparición del dolor por regeneración nerviosa, pudiendo repetir la rizolisis. Algunos predictores de mala respuesta son la depresión, el síndrome de cirugía fallida y la afectación de múltiples niveles; debiendo tener estos factores en cuenta a la hora de indicar el procedimiento ${ }^{14,38-42}$.

\section{Conclusión}

La rizolisis de la rama medial del ramo dorsal del nervio espinal es la técnica de elección en el tratamiento del síndrome facetario lumbar, superior a otros procedimientos intervencionistas como la infiltración de esteroides intra articular y a tratamientos conservadores. La realización de la técnica con TC

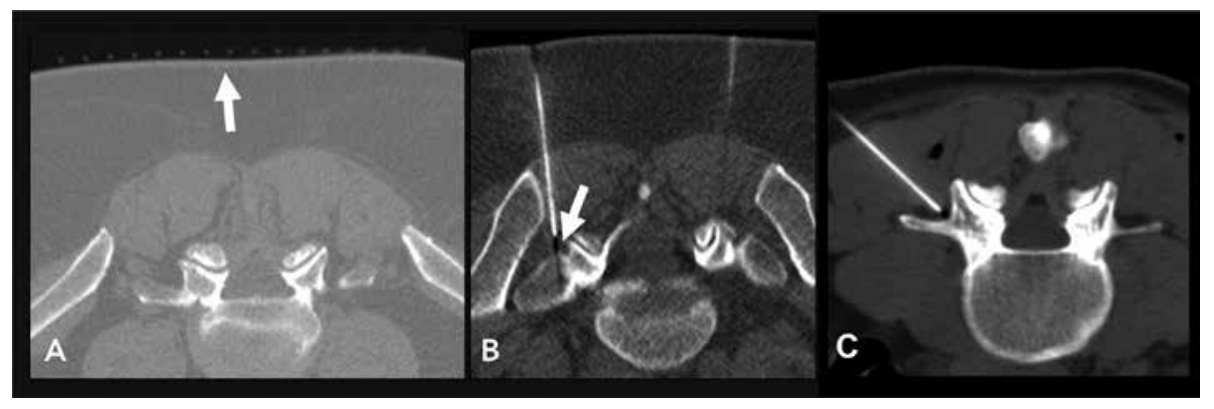

Figura 7. A) Imagen axial de TC en la que se aprecia rejilla radio opaca para guiar la punción (flecha). B) Imagen axial de TC que muestra la adecuada localización de la aguja (flecha) en el nivel vertebral S1. C) Imagen axial de TC con abordaje oblicuo de la aguja en L5 para un mayor contacto del electrodo con el nervio. 
permite una gran precisión en la punción incluso en operadores con menor experiencia, aunque aún no se ha demostrado su superioridad frente a la guía radioscópica.

El bloqueo nervioso de la rama medial del ramo dorsal del nervio espinal es un paso previo indispensable a la realización de la rizolisis, determinando en gran parte la probabilidad de éxito del procedimiento.

\section{Bibliografía}

1. Manchikanti L, Singh V, Falco FJ, Benyamin RM, Hirsch JA. Epidemiology of low back pain in adults. Neuromodulation 2014; 17 Suppl 2: 3-10.

2. Medel-Rebollo J, Ribera-Canudas MV, Mesas Idáñez A, Márquez-Martínez E, Martínez-Ripol P, CandelaCustardoy $A$, et al. Técnicas mínimamente invasivas en el tratamiento del dolor crónico. Semin Fund Esp Reumatol 2013; 14: 135-41.

3. Amirdelfan K, McRoberts P, Deer TR. The differential diagnosis of low back pain: a primer on the evolving paradigm. Neuromodulation 2014; 17 Suppl 2: 11-17.

4. Webster LR, Markman J. Medical management of chronic low back pain: efficacy and outcomes. Neuromodulation 2014; 17 Suppl 2: 18-23.

5. Wellington J. Noninvasive and alternative management of chronic low back pain (efficacy and outcomes). Neuromodulation 2014; 17 Suppl 2: 24-30.

6. Misaggi B, Gallazzi M, Colombo M, Ferraro M. Articular facets syndrome: diagnostic grading and treatment options. Eur Spine J 2009; 18 Suppl 1: 49-51.

7. Golovac S. Radiofrequency Neurolysis. Neuroimaging Clin N Am 2010; 20 (2): 203-214.

8. Masala S, Nano G, Mammucari M, Marcia S, Simonetti G. Medial branch neurotomy in low back pain. Neuroradiology 2012; 54(7): 737-744.

9. Roy C, Chatterjee N. Medial branch neurotomy in low back pain. Neuroradiology 2012; 54(7): 781-782; author reply 783 .

10. Civelek E, Cansever T, Kabatas S, Kircelli A, Yilmaz C, Musluman M, et al. Comparison of effectiveness of facet joint injection and radiofrequency denervation in chronic low back pain. Turk Neurosurg 2012; 22(2): 200-206.

11. Van Zundert J, Vanelderen $P$, Kessels A, van Kleef $M$. Radiofrequency treatment of facet-related pain: evidence and controversies. Curr Pain Headache Rep 2012; 16(1): 19-25.

12. Dreyfuss P, Halbrook B, Pauza K, Joshi A, McLarty $J$, Bogduk N. Efficacy and validity of radiofrequency neurotomy for chronic lumbar zygapophysial joint pain. Spine 2000; 25(10): 1270-1277.

13. Markman JD, Philip A. Interventional approaches to pain management. Med Clin North Am 2007; 91(2): 271-286.

14. Manchikanti L, Abdi S, Atluri S, Benyamin RM, Boswell MV, Buenaventura RM, et al. An update of comprehensive evidence-based guidelines for interventional techniques in chronic spinal pain. Part II: guidance and recommendations. Pain Physician 2013; 16(2 Suppl): S49-S283.

15. Sae-Jung S, Jirarattanaphochai K. Outcomes of lumbar facet syndrome treated with oral diclofenac or methylprednisolone facet injection: a randomized trial.
Int Orthop. 2016. [Epub ahead of print].

16. Baron R, Binder A, Attal N, Casale R, Dickenson $\mathrm{AH}$, Treede RD. Neuropathic low back pain in clinical practice. Eur J Pain. 2016. [Epub ahead of print].

17. Kalichman L, Li L, Kim DH, Guermazi A, Berkin V, O'Donnell CJ, et al. Facet joint osteoarthritis and low back pain in the community- based population. Spine (Phila Pa 1976) 2008; 33: 2560-2565.

18. Tibrewal S, Khan OH, Tibrewal SB. Facet joint injection in lower back pain--is its continued use justified?. J R Soc Med 2007; 100(7): 301-302.

19. Rees WS. Letter: Subcutaneous lumbar rhizolysis. Med J Aust 1974; 1(11): 415.

20. Rees WS. Rhysolysis of the nerves of the zygoapophyseal joints. Spine (Phila Pa 1976) 1983; 8(1): 118-120.

21. Carette S, Marcoux S, Truchon R, Grondin C, Gagnon $\mathrm{J}$, Allard $\mathrm{Y}$, et al. A controlled trial of corticosteroid injections into facet joints for chronic low back pain. $\mathrm{N}$ Engl J Med 1991; 325(14): 1002-1007.

22. Jackson RP, Jacobs RR, Montesano PX. 1988 Volvo award in clinical sciences. Facet joint injection in lowback pain. A prospective statistical study. Spine (Phila Pa 1976) 1988; 13(9): 966-971.

23. Manchikanti L, Singh V. Diagnosis of facet joint pain and prediction of success and failure for cervical facet radiofrequency denervation. Reg Anesth Pain Med 2009; 34(1): 81-82.

24. Manchikanti L, Pampati S, Cash KA. Making sense of the accuracy of diagnostic lumbar facet joint nerve blocks: an assessment of the implications of $50 \%$ relief, $80 \%$ relief, single block, or controlled diagnostic blocks. Pain Physician 2010; 13(2): 133-143.

25. Falco FJ, Manchikanti L, Datta S, Sehgal N, Geffert S, Onyewu O, et al. An update of the systematic assessment of the diagnostic accuracy of lumbar facet joint nerve blocks. Pain Physician 2012; 15(6): E869-907.

26. Falco FJ, Manchikanti L, Datta S, Sehgal N, Geffert $\mathrm{S}$, Onyewu $\mathrm{O}$, et al. An update of the effectiveness of therapeutic lumbar facet joint interventions. Pain Physician 2012; 15(6): E909-953.

27. Son BC, Kim HS, Kim IS, Yang SH, Lee SW. Percutaneous radiofrequency thermocoagulation under fluoroscopic image-guidance for idiopathic trigeminal neuralgia. $J$ Korean Neurosurg Soc 2011; 50(5): 446-452.

28. Plasencia Martínez JM. Pulmonary radiofrequency ablation (Part 1): current state. Radiologia 2015; 57(4): 275-286.

29. Soloman M, Mekhail MN, Mekhail N. Radiofrequency Treatment in Chronic Pain. Expert Rev Neurother 2010; 10(3): 469-474.

30. Veizi E, Hayek S. Interventional therapies for chronic low back pain. Neuromodulation 2014; 17 Suppl 2: 31-45.

31. Colini-Baldeschi G. Evaluation of pulsed radiofrequency denervation in the treatment of chronic facetjoint pain: an observational study. Anesth Pain Med 2012; 1(3): 168-173.

32. Koizuka S, Saito S, Kawauchi C, Takizawa D, Goto F. Percutaneous radiofrequency lumbar facet rhizotomy guided by computed tomography fluoroscopy. J Anesth 2005; 19: 167-169.

33. Galiano K, Obwegeser AA, Bodner G, Freund M, Maurer $\mathrm{H}$, Kamelger FS, et al. Ultrasound guidance for facet joint injections in the lumbar spine: Acomputed 
tomography-controlled feasibility study. Anesth Analg 2005; 101: 579-583.

34. Wu T, Zhao WH, Dong Y, Song HX, Li JH. Effectiveness of Ultrasound-Guided Versus Fluoroscopy or Computed Tomography Scanning Guidance in Lumbar Facet Joint Injections in Adults With Facet Joint Syndrome: A Meta-Analysis of Controlled Trials. Arch Phys Med Rehabil 2015 Dec. Epub ahead of print.

35. Park CH. Comprasion of Effectiveness of CT vs C-arm Guided Percutaneous Radiofrequency Lumbar Facet Rhizotomy. Korean J Pain 2010; 23: 137-141.

36. Pacetti M, Fiaschi P, Gennaro S. Percutaneous radiofrequency thermocoagulation of dorsal ramus branches as a treatment of "lumbar facet syndrome"--How I do it. Acta Neurochir (Wien) 2016; 158: 995-998.

37. Pérez-Cajaraville J, Sancho-De Ávila A, Cabrera I, Abejón D. Radiofrecuencia de facetas lumbares y cervicales. Rev Soc Esp Dolor 2011; 18: 249-258.

38. Nagda JV, Davis CW, Bajwa ZH, Simopoulos TT. Retrospective review of the efficacy and safety of repeated pulsed and continuous radiofrequency lesioning of the dorsal root ganglion/segmental nerve for lumbar radicular pain. Pain Physician 2011; 14: 371-376.

39. Cohen SP, Bajwa ZH, Kraemer JJ, Dragovich A, Williams $\mathrm{KA}$, Stream J, et al. Factors predicting success and failure for cervical facet radiofrequency denervation: a multi-center analysis. Reg Anesth Pain Med 2007; 32: 495-503.

40. Cohen SP, Hurley RW, Christo PJ, Winkley J, Mohiuddin MM, Stojanovic MP. Clinical predictors of success and failure for lumbar facet radiofrequency denervation. Clin J Pain 2007; 23: 45-52.

41. Streitberger K, Müller T, Eichenberger U, Trelle S, Curatolo M. Factors determining the success of radiofrequency denervation in lumbar facet joint pain: a prospective study. Eur Spine J 2011; 20: 2160-2165.

42. McCormick ZL, Marshall B, Walker J, McCarthy R, Walega DR. Long-Term Function, Pain and Medication Use Outcomes of Radiofrequency Ablation for Lumbar Facet Syndrome. Int J Anesth Anesth 2015; 2. 